\title{
Design of Multiplierless Correlators for Timing Synchronization in IEEE 802.11a Wireless LANs
}

\author{
Kun-Wah Yip, Member, IEEE, Yik-Chung Wu, Student Member, IEEE, \\ and Tung-Sang Ng, Fellow, IEEE
}

\begin{abstract}
Timing synchronization for IEEE 802.11a WLANs requires using a correlator to correlate the received signal with a known waveform. Straightforward implementation of this correlator results in the need to perform 320 million complex multiplications per second. This significant requirement can be eliminated by using multiplierless correlators. In this paper, multiplierless correlators are designed based on constraining the real and imaginary parts of correlator coefficients to be sums of powers of two. Sets of coefficients that yield good synchronization performance for simple AWGN channels are first identified; then their goodness for indoor communication environments is verified by simulation for multipath fading channels. Several multiplierless correlators are found. Comparison among these correlators identifies a good one that requires to perform only 26 addition/subtraction operations per correlator output while a similar synchronization performance can be maintained. ${ }^{\prime}$

Index Terms - Low-complexity implementation, Multiplierless correlator, Timing synchronization, Wireless local area network.
\end{abstract}

\section{INTRODUCTION}

$I^{\mathrm{E}}$ EEE 802.11a wireless local area networks (WLANs) support packetized transmission at a high data rate up to $54 \mathrm{Mbps}$ [1]. Orthogonal frequency division multiplexing (OFDM) is used as the modulation technique. Before data can be demodulated, OFDM frames need to be time-synchronized. Since a sequence of known OFDM symbols is embedded into the preamble of each data packet, timing synchronization can be achieved by detecting the end time of this sequence. To detect the end time, the receiver can use a sliding correlator to correlate the received signal with the waveform of a known OFDM symbol in the preamble. Implementation of this correlator, however, involves significant complexity. It will be shown that straightforward implementation of this correlator results in the need to perform 320 million complex

\footnotetext{
${ }^{1}$ This work was supported by the Hong Kong Research Grants Council and by the University Research Committee of The University of Hong Kong, Hong Kong.

K.-W. Yip and T.S. Ng are with the Department of Electrical and Electronic Engineering, The University of Hong Kong, Pokfulam Road, Hong Kong. Phone: (852) 2857 8406. Fax: (852) 2559 8738. (Email: kwyip@eee.hku.hk; tsng@eee.hku.hk)

Y.C. Wu was with the Department of Electrical and Electronic Engineering, The University of Hong Kong. He is now with the Department of Electrical Engineering, Texas A\&M University, College Station, TX 77843-3128. Phone: (979) 8628000 . Fax: (979) 862 4630. (Email: ycwu@ee.tamu.edu)
}

Contributed Paper

Original manuscript received January 24, 2003

Revised manuscript received January 27, 2003

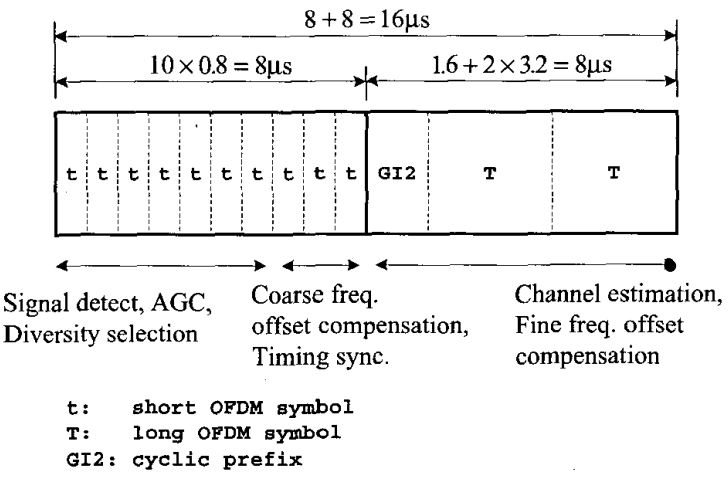

Fig. 1. Preamble structure of an IEEE 802.11a data packet.

multiplications per second. In this paper, correlators that eliminate the need to perform multiplication are designed. Several multiplierless correlators are found. In particular, comparison among these correlators identifies a good one that requires to perform only 26 addition/subtraction operations per correlator output while a similar synchronization performance can be maintained. Using this multiplierless correlator leads to considerable reduction in the implementation complexity of receivers. The advantage to implement IEEE 802.11 a WLAN receivers at a lower complexity (and hence a lower cost) is of significance in the field of consumer electronics as one emerging application of WLANs is the in-house distribution of audio-visual signals [2], [3].

This paper is organized as follows. Section II provides a background on the timing-synchronization aspects for IEEE 802.11a WLANs. The design of multiplierless correlators is carried out in Section III. Conclusions are given in Section IV. Several appendices follow. A mathematical model of the timing-synchronization procedure is given in Appendix I. This model is used in Appendix II to analyze the timingsynchronization performance for AWGN channels. The result is used in the design of multiplierless correlators. Appendix III details the simulation conditions under which the goodness of the multiplierless correlators designed here is evaluated.

\section{TIMING SYNCHRONIZATION IN IEEE 802.11A WLANS}

A $16 \mu$ s preamble having a structure shown in Fig. 1 is inserted at the beginning of each data packet. The preamble is divided into two subpreambles. The first one consists of ten identical, short OFDM symbols each having a length of $800 \mathrm{~ns}$. The second one comprises two long OFDM symbols each of 
length $3.2 \mu$ s preceded by a $1.6 \mu$ s cyclic prefix. The first subpreamble is used for initial detection of the signal, automatic gain control, diversity selection, coarse frequencyoffset compensation and timing synchronization. The second one enables channel estimation and fine frequency-offset compensation.

It is known that frequency-offset estimation can be accomplished by performing two FFTs on the portion of signal comprising two repeated symbols and then measuring the phase rotation [4]. Since the first subpreamble is a repetition of identical symbols, the signal is periodic with a period of $800 \mathrm{~ns}$. The receiver can choose any $1.6 \mu \mathrm{s}$ window to perform FFTs and needs not know the exact timing of symbol boundaries. On the other hand, it is required to detect the end of the first subpreamble. This time reference enables the receiver to locate the time instant in the second subpreamble at which the FFT window for fine frequency-offset compensation begins, and the starting time instant for data demodulation of OFDM symbols. To detect the end of the first subpreamble, the receiver can correlate the received signal with the short OFDM symbol. The presence of a correlation peak indicates that the first subpreamble has not passed while the absence of an expected correlation peak is an indication that the current time position is at $800 \mathrm{~ns}$ in the second subpreamble. A sliding correlator, which produces correlation results at a rate equal to the rate of incoming signal samples, is required because the receiver needs to perform rapid synchronization in a short time. A sampling rate of $20 \mathrm{MHz}$ is recommended in the digital implementation of the correlator. ${ }^{2}$ Note that both coarse frequency-offset compensation and timing synchronization are carried out simultaneously. A large frequency offset may be present during correlation. Despite this, it is shown in [5] that this frequency offset does not normally have a significant effect on the timingsynchronization performance.

\section{DESIGN OF MULTIPLIERLESS CORRELATORS}

The sliding correlator used for timing synchronization correlates the received signal samples with the short OFDM symbol. Since the rate of the received signal samples is $20 \mathrm{MHz}$ and the short OFDM symbol has a length of $800 \mathrm{~ns}$, it follows that 16 signal samples are respectively multiplied with 16 correlator coefficients to generate one correlator output. Let $\left\{r_{n}\right\}$ be the sequence of complex-valued received signal samples ${ }^{3}$, and $\left\{\Xi_{n}\right\}$ be the sequence of (complex-valued) correlator outputs obtained at the same rate of the received signal samples. The correlation equation is given by

$\Xi_{n}=\sum_{m=1}^{16} r_{n-16+m} g_{m}^{*}$

2 This sampling rate is mentioned in [1] for the implementation of OFDM demodulators.

3 Each signal sample has in-phase and quadrature-phase components of the received signal, so it is complex-valued.
TABLE 1.

CORRELATOR COEFFICIENTS $\left(\widetilde{g}_{m}\right)$ SELECTED AS THE SAMPLED VALUES OF THE SHORT OFDM WAVEFORM.

\begin{tabular}{cr}
\multicolumn{3}{c}{ THE SHORT OFDM WAVEFORM. } \\
\hline$m$ & $-1.18+\tilde{g}_{m}$ \\
\hline 1 & $-0.12-0.02 j$ \\
3 & $1.27-0.11 j$ \\
4 & 0.82 \\
5 & $1.27-0.11 j$ \\
6 & $-0.12-0.70 j$ \\
7 & $-1.18+0.02 j$ \\
8 & $0.41+0.41 j$ \\
9 & $0.02-1.18 j$ \\
10 & $-0.70-0.12 j$ \\
11 & $-0.11+1.27 j$ \\
12 & $-0.11+1.27 j$ \\
13 & $-0.70-0.12 j$ \\
14 & $0.02-1.18 j$ \\
15 & $0.41+0.41 j$ \\
16 &
\end{tabular}

where $g_{m}$ is the $m$ th correlator coefficient.

A straightforward choice of correlator coefficients is the sequence of sampled values of the short OFDM symbol. For the sake of clarity, we denote the $m$ th coefficient for this choice as $\tilde{g}_{m}$. The coefficients are listed in Table 1 , computed by

$\widetilde{g}_{m}=\sum_{k=-26}^{26} B_{k} e^{j 2 \pi k \Delta_{f} m T_{s a m}}, \quad m=1,2, \cdots, 16$,

where $T_{\text {sam }}=50 \mathrm{~ns}$ is the sampling interval, and the parameters $B_{k}$ and $\Delta_{f}$ are given in Appendix I. $^{4}$ It is apparent that these coefficients are in general complex-valued. Since the correlator generates its outputs at a rate of $20 \mathrm{MHz}$, and since 16 complex multiplications are involved in the generation of one output, the correlator that employs $\widetilde{g}_{m}$ 's needs to perform 320 million complex multiplications per second. This significant requirement can be eliminated by using multiplierless correlators that are developed as follows.

It is easy to show that if the real and imaginary parts of the correlator coefficients can be expressed as sums of powers of two (i.e., in the form $\sum_{n} 2^{-n}$ where the $n$ values are integers), only shifting and addition operations are required in the implementation so that the need to perform multiplication can be removed. We impose the constraint that the real and imaginary parts of $g_{m}$ satisfy

$$
\begin{aligned}
\operatorname{Re}\left(g_{m}\right) & \operatorname{Im}\left(g_{m}\right) \\
\in & \{-1,-1+\Delta,-1+2 \Delta, \cdots, 0, \cdots, 1-2 \Delta, 1-\Delta, 1\}
\end{aligned}
$$

where the quantization step size $\Delta$ is given by $\Delta=2^{-n}, n$

4 Note that the data listed in Table 1 differ from the reference data shown in Table G.4 of [1] only by a scale factor. This difference does not affect the development of multiplierless correlators in this work. 
TABLE 2.

CORRELATOR COEFFICIENTS $\left(g_{m}\right)$ FOR MULTIPLIERLESS CORRELATORS THAT ARE FOUND TO ACHIEVE GOOD SYNCHRONIZATION PERFORMANCE FOR AWGN CHANNELS.

\begin{tabular}{|c|c|c|c|c|c|}
\hline \multirow{2}{*}{$\frac{m}{1}$} & \multicolumn{2}{|c|}{$\Delta=0.25$} & \multicolumn{2}{|c|}{$\begin{array}{c}g_{m} \\
\Delta=0.5\end{array}$} & $\Delta=1$ \\
\hline & -1 & & -1 & & -1 \\
\hline 2 & & $-0.5 j$ & & $-0.5 j$ & $-1 j$ \\
\hline 3 & 1. & & 1 & & $I$ \\
\hline 4 & 0.75 & & 1 & & 1 \\
\hline 5 & 1 & & 1 & & 1 \\
\hline 6 & & $-0.5 j$ & & $-0.5 j$ & $-1 j$ \\
\hline 7 & -1 & & -1 & & -1 \\
\hline 8 & 0.25 & $+0.25 j$ & 0.5 & $+0.5 j$ & 0 \\
\hline 9 & & $-1 j$ & & $-1 j$ & $-1 j$ \\
\hline 10 & -0.5 & & -0.5 & & -1 \\
\hline 11 & & $+1 j$ & & $+1 j$ & $\cdot+1 j$ \\
\hline 12 & & $+0.75 j$ & & $+1 j$ & $+1 j$ \\
\hline 13 & & $+1 j$ & & $+1 j$ & $+1 j$ \\
\hline 14 & -0.5 & & -0.5 & & -1 \\
\hline 15 & & $-1 j$ & & $-1 j$ & $-1 j$ \\
\hline 16 & 0.25 & $+0.25 j$ & 0.5 & $+0.5 j$ & 0 \\
\hline
\end{tabular}

being a non-negative integer. For instance, this set is $\{-1$, $-0.5,0,0.5,1\}$ if $\Delta=0.5$. It is desirable to select a set of $g_{m}$ 's that can achieve the best synchronization performance. However, determination of this optimal set requires exhaustive search, which is computationally prohibitive especially when $\Delta$ is small. To reduce the effort to a manageable level, we adopt the following two-step approach. We first identify sets of correlator coefficients that yield good synchronization performance in terms of the probability of synchronization failure, $P_{\text {fail }}$, for simple AWGN channels. Considering AWGN channels is because the expression to compute $P_{\text {fail }}$ is available, and computing $P_{\text {fail }}$ based on the formula is computationally much less demanding than obtaining it via simulation. Hence, identifying good sets of coefficients can be accomplished quickly. The second step is to verify the goodness of these sets for typical indoor communication environments by simulating the synchronization performance for multipath fading channels.

The correlator coefficients are determined by

$$
\begin{array}{r}
g_{m}=\text { quan }\left\{c \times \operatorname{Re}\left(\tilde{g}_{m}\right)\right\}+j \cdot \text { quan }\left\{c \times \operatorname{Im}\left(\tilde{g}_{m}\right)\right\}, \\
m=1,2, \cdots, 16,
\end{array}
$$

where $\widetilde{g}_{m}$ is given by (2), $c$ is a scale constant the same for all $m$ and is to be determined, and quan $(x)$ is the quantization function that maps the real number $x$ into an allowable value specified by (3). It follows that

$$
\text { quan }(x)=\left\{\begin{array}{ll}
-1 & \text { if } x<-1+\frac{\Delta}{2} \\
-1+k \Delta & \text { if }-1+\frac{\Delta}{2}+(k-1) \Delta \leq x<-1+\frac{\Delta}{2}+k \Delta \\
1 & \text { if } 1-\frac{\Delta}{2} \leq x
\end{array} \quad \text { where } k=1,2, \cdots, \frac{2}{\Delta}-1\right.
$$

We select the value of $c$ that minimizes $P_{\text {fail }}$ for AWGN channels. The expression of $P_{\text {fail }}$ is derived in Appendix II and is given by (29). Then the corresponding set of $g_{m}$ 's is obtained by (4). The sets of correlator coefficients obtained by this method for $^{5} \in\{0.25,0.5,1\}$ are listed in Table 2 . The corresponding implementation equations, obtained by expanding (1) with the respective sets of $g_{m}$ 's, are listed in Table 3.

The goodness of these sets are verified by simulating $P_{\text {fail }}$ for multipath Rician fading channels, which are appropriate to model the characteristic of indoor radio channels [6]-[8]. A multipath Rician-fading channel is characterized by its Rician factor, $K$, and the RMS delay spread for the Rayleigh-fading component, $\tau_{r m s}$. The simulation conditions are detailed in Appendix III. Fig. 2 plots $P_{\text {fail }}$ against $E_{d s} / N_{0}$ for sets of $g_{m}$ 's shown in Table 2 under the conditions that $\tau_{r m s}=50 \mathrm{~ns}$ and $K \in\{-\infty \mathrm{dB}, 6 \mathrm{~dB}\}$. (Note that when $K=-\infty \mathrm{dB}$, the channel becomes a Rayleigh-fading channel.) The $P_{\text {fail }}$ curve for the case that employs $\tilde{g}_{m}$ 's is also plotted as a reference case for comparison. Each $P_{\text {fail }}$ value was obtained by performing $10^{6}$ simulation runs, except when $E_{d s} / N_{0} \geq 20 \mathrm{~dB}$ and $K=6 \mathrm{~dB}$, where $10^{7}$ simulation runs were used to achieve

5 Under the condition that $E_{d s} / N_{0}=10 \mathrm{~dB}$ in the calculation of $P_{\text {fail }}$ by (29). 
TABLE 3.

IMPLEMENTATION EQUATIONS FOR THE MULTIPLIERLESS CORRELATORS BASED ON COEFFICIENTS SHOWN IN TABLE 2.

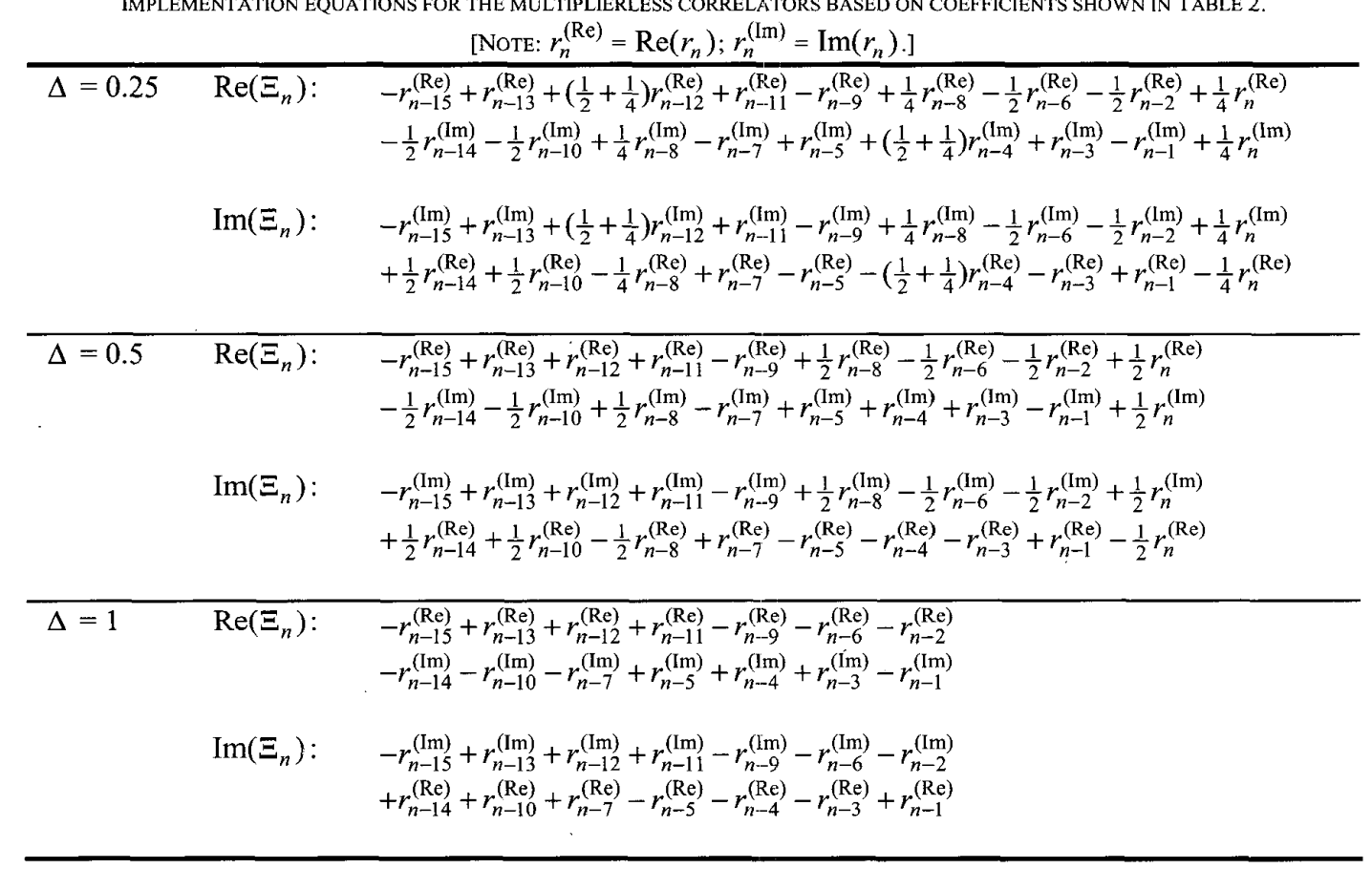

better accuracy. It is apparent from Fig. 2 that multiplierless correlators using $g_{m}$ 's for $\Delta=0.25$ and $\Delta=1$ results in a synchronization performance close to, and most often slightly better than, that achieved by a non-multiplierless correlator using $\widetilde{g}_{m}$ 's. For the case of $\Delta=0.5$, the performance is slightly worse than that of the reference case. Therefore, it is desirable to use the sets of correlator coefficients with $\Delta=$ 0.25 and $\Delta=1$.

Although both sets of correlator coefficients developed under the conditions of $\Delta=0.25$ and $\Delta=1$ yield similar synchronization performance, a correlator with $\Delta=1$ is preferred since it is less complex in the implementation. This advantage is illustrated in Table 4 , which lists the numbers of two-input addition/subtraction operations on binary numbers (not complex-valued numbers) required in the implementation of multiplierless correlators for different $\Delta$. It is apparent that for the case of $\Delta=1$, only 26 two-input addition/subtraction calculations are involved in the generation of one correlator output. In comparison with the case of $\Delta=0.25$, which requires to perform 38 such calculations in generating one correlator output, a saving of $(38-26) / 38=32 \%$ in the implementation complexity can be obtained.

\section{CONCLUSIONS}

In this paper, we have designed multiplierless correlators that can be used for low-complexity implementation of timing- synchronization circuits in IEEE 802.11a WLANs. It has been found that the correlators developed under the conditions of $\Delta$ $=0.25$ and $\Delta=1$ attain synchronization performances close to that achieved by a non-multiplierless correlator using a straightforward choice of correlator coefficients. It follows that using the developed multiplierless correlators achieve a similar synchronization performance while a considerable reduction in the implementation complexity can be obtained. For the two multiplierless correlators with $\Delta=0.25$ and $\Delta=$ 1 , it has been shown that the one using $\Delta=1$ requires less number of addition/subtraction operations in generating correlator outputs than the other one does. In practical implementation, it is therefore preferred to use the multiplierless correlator with $\Delta=1$, where the implementation equations are listed in the last row of Table 3 and only 26 addition/subtraction operations are required to compute one correlation output.

\section{APPENDIX I. MATHEMATICAL MODEL OF THE TIMING- SYNCHRONIZATION PROCEDURE}

The OFDM signal specified in the standard [1] comprises 52 subcarriers numbered from -26 to +26 with no signal transmission at the zeroth subcarrier. All subcarriers are utilized to generate the long OFDM symbol. For the short one, transmission is confined to the subcarriers whose numbers are multiples of 4 , so that only 12 subcarriers are used. The complex envelope of the preamble, $s(t)$, is given by [1] 


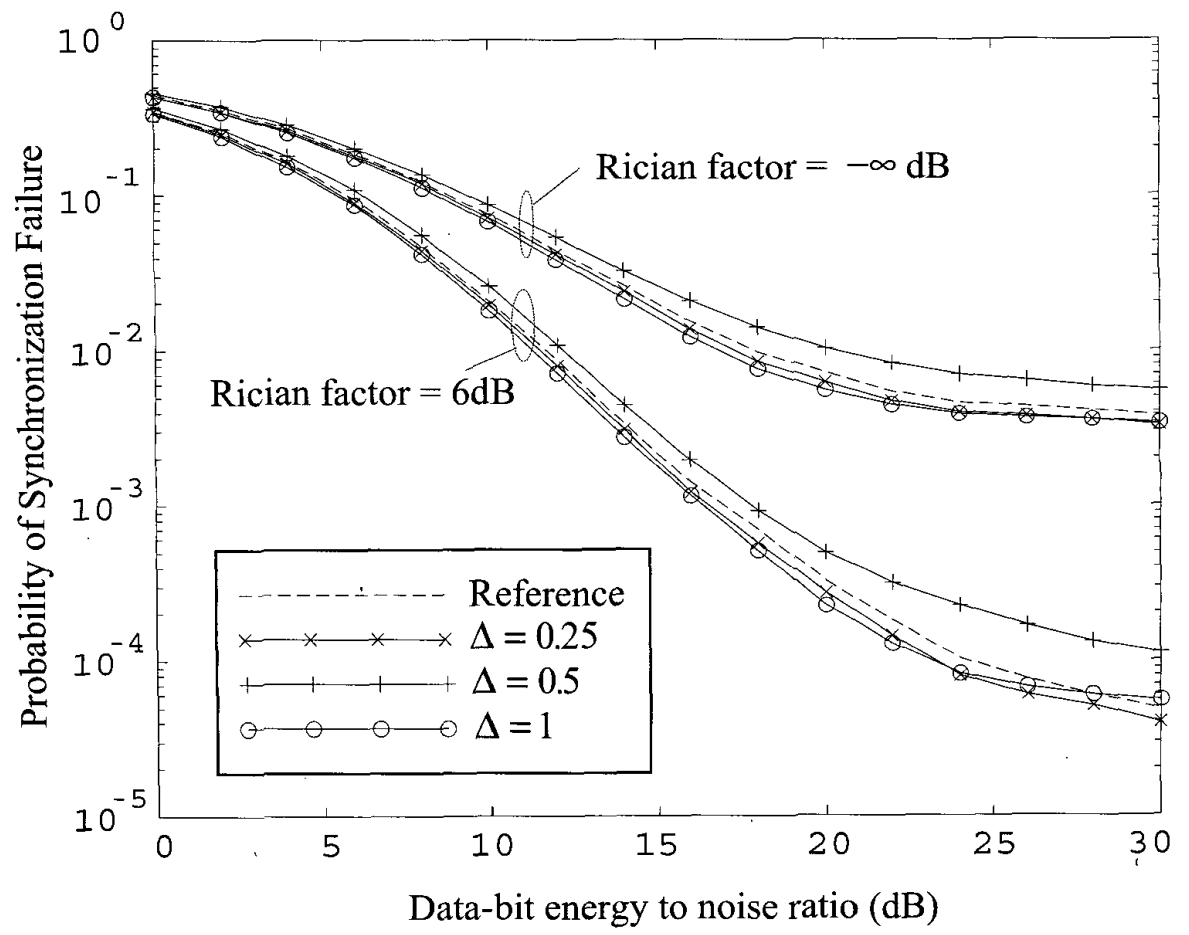

Fig. 2. $P_{\text {fail }}$ against $E_{d s} / N_{0}$ for correlators using different sets of coefficients.

TABLE 4.

NUMBER OF TWO-INPUT ADDITION/SUBTRACTION OPERATIONS REQUIRED TO GENERATE ONE CORRELATOR OUTPUT FOR MULTIPLIERLESS CORRELATORS OF DIFFERENT QUANTIZATION STEP SIZE $\Delta$.

Number of operations required for:

\begin{tabular}{lll}
$\Delta=0.25 \quad \Delta=0.5 \quad \Delta=1$ \\
\hline
\end{tabular}

38

34

26

$$
\begin{aligned}
s(t) & =\sqrt{2 P} \times\left\{\sum_{k=-26}^{26} B_{k} e^{j 2 \pi k \Delta_{f} t} w\left(0, T_{p 1}\right)\right. \\
& \left.+\sum_{k=-26}^{26} C_{k} e^{j 2 \pi k \Delta_{f}\left(t-T_{p 1}-T_{G I 2}\right)} w\left(T_{p 1}, T_{p 1}+T_{p 2}\right)\right\}
\end{aligned}
$$

where (a) $T_{p 1}=8 \mu \mathrm{s}$ and $T_{p 2}=8 \mu \mathrm{s}$ are the lengths of the first and second subpreambles, respectively, (b) $T_{G I 2}=1.6 \mu \mathrm{s}$ is the length of the cyclic prefix of the second subpreamble, (c) $w\left(t_{1}, t_{2}\right)$ is the raised-cosine window given by $w\left(t_{1}, t_{2}\right)=$
$\left\{\begin{array}{cc}\sin ^{2}\left(\frac{\pi}{2}\left[0.5+\left(t-t_{1}\right) / T_{T R}\right]\right), & t_{1}-T_{T R} / 2 \leq t<t_{1}+T_{T R} / 2 \\ 1, & t_{1}+T_{T R} / 2 \leq t<t_{2}-T_{T R} / 2 \\ \sin ^{2}\left(\frac{\pi}{2}\left[0.5+\left(t-t_{2}\right) / T_{T R}\right]\right), & t_{2}-T_{T R} / 2 \leq t<t_{2}+T_{T R} / 2 \\ 0, & \text { otherwise }\end{array}\right.$

wherein $T_{T R}=100 \mathrm{~ns}$, (d) $P$ is the signal power, (e) $\Delta_{f}=$ $312.5 \mathrm{kHz}$ is the subcarrier spacing, and (f) $B_{k}=52^{-1 / 2} S_{k}$ and $C_{k}=52^{-1 / 2} L_{k}$ are pilot symbols used to generate the short and long OFDM symbols, respectively, wherein $S_{k}$ and $L_{k}$ are given by Eqris. (6) and (8), respectively, of [1]. We mention that $B_{0}=C_{0}=0$, and $B_{k}=0$ for $k$ not a multiple of 4 .

The complex envelope of the received signal after RF filtering, $r(t)$, is given by

$r(t)=\eta(t)+(h(t) \otimes s(t))$

where $\otimes$ denotes convolution, $\eta(t)$ is the filtered complexGaussian noise, and $h(t)$ is the channel impulse response. The noise power is computed by

$\frac{1}{2} E\left\{|\eta(t)|^{2}\right\}=N_{0} W$ 
where $N_{0}$ is the one-sided noise power spectral density of additive white Gaussian noise and $W$ is the noise bandwidth of the RF filter. Since the signal energy is concentrated within a bandwidth of $18 \mathrm{MHz}$ [1], we consider that $W=18 \mathrm{MHz}$. The received signal is digitized at a sampling rate of $1 / T_{\text {sam }}$ samples per second with $T_{\text {sam }}^{-1}=20 \mathrm{MHz}$. The $n$th signal sample, $r_{n}$, is given by

$r_{n}=r\left(n T_{\text {sam }}+\tau_{\text {sam }}\right)$

where $\tau_{\text {sam }}$ is the fractional time offset modeled by a uniform random variable over $\left[-\frac{1}{2} T_{\text {sam }}, \frac{1}{2} T_{\text {sam }}\right]$. It is possible that the noise components of $r_{n}$ 's are correlated where the correlation depends on the frequency response of the RF filter and the sampling rate. Since the filter bandwidth is approximately $20 \mathrm{MHz}$ and the sampling rate is also $20 \mathrm{MHz}$, it is assumed that this correlation is negligible.

A sliding correlator is used to process the received signal samples. We denote $T_{s s}=800 \mathrm{~ns}$ as the length of the short OFDM symbol. The correlator output obtained at time $n T_{\text {sam }}$ $+\tau_{\text {sam }}$ is computed by

$\Xi_{n}=\sum_{m=1}^{M} r_{n-M+m} g_{m}^{*}$

where

$M=T_{s s} / T_{\text {sam }}=16$

is the number of signal samples involved in correlation, and the $g_{m}, m=1,2, \cdots, M$, are the correlator coefficients that constitute a short OFDM symbol. [Note that (1) is reproduced here as (11).] The synchronization procedure is as follows. The time location of the correlation peak and its $\left|\Xi_{n}\right|^{2}$ value are identified by searching for the maximum value of $\left|\Xi_{n}\right|^{2}$ over the obtained correlation results. After the correlation peak is located, the next $M$ th correlation result is compared with the maximum value of $\left|\Xi_{n}\right|^{2}$ to determine if another correlation peak is present. The search for expected correlation peaks continues until an absence is identified, in which the second subpreamble is entered. Note that a new correlation result is generated every sampling instant so that the maximum value of $\left|\Xi_{n}\right|^{2}$ and the corresponding time location are regularly updated. Let $\mu_{i}$ and $n_{i}$ be the maximum value of $\left|\Xi_{n}\right|^{2}$ and the corresponding time location, respectively, recorded at the $i$ th sampling instant but excluding the current correlation result. They are given by

$\mu_{i}=\max _{n<i}\left|\Xi_{n}\right|^{2}$

and $n_{i}=\underset{n<i}{\arg \max }\left|\Xi_{n}\right|^{2}$

The end of the first subpreamble is declared at the sampling instant $i-M$ if $n-n_{i}$ is a multiple of $M$ and the condition

$\beta \mu_{i}>\left|\Xi_{i}\right|^{2}$

is satisfied, where $\beta \in(0,1)$ is a given threshold value. In this paper, we adopt $\beta=0.5$ [5] in the design of multiplierless correlators.

\section{APPENDIX II. PROBABILITY OF SYNCHRONIZATION FAILURE FOR AN AWGN CHANNEL}

In the analysis we follow the suggestion of the specifications [1] that the last three short OFDM symbols are used for timing synchronization, although in practice it may begin earlier or later. It follows that correlation peaks occur at $n=8 M, 9 M$ and $10 \mathrm{M}$. At $n=11 \mathrm{M}$, the value of $\left|\Xi_{n}\right|^{2}$ drops significantly. An event of false alarm arises when (15) is satisfied for $n=9 M$ or $10 M$. A miss occurs when (15) is not satisfied for $n=11 M$. A synchronization failure is an event where either a false alarm or a miss occurs.

We consider an AWGN channel so that the channel impulse response is given by $h(t)=1$. For simplicity in the derivation, we assume that a rectangular window instead of a raisedcosine one is used in (6). Expanding (10) by (8) and substituting the resultant expression into (11) yields

$\Xi_{q M}=\left\{\begin{array}{cc}\sqrt{2 P} \times\left[\eta_{q M}+Y\left(\tau_{\text {sam }}\right)\right] & q=8,9,10 \\ \sqrt{2 P} \times\left[\eta_{q M}+Z\left(\tau_{\text {sam }}\right)\right] & q=11\end{array}\right.$

where

$Y\left(\tau_{s a m}\right)=\sum_{m=1}^{M} g_{m}^{*} \sum_{k=-26}^{26} B_{k} e^{j 2 \pi k \Delta_{f}\left(m T_{s a m}+\tau_{s a m}\right)}$,
$Z\left(\tau_{s a m}\right)=\sum_{m=1}^{M} g_{m}^{*} \sum_{k=-26}^{26} C_{k} e^{j 2 \pi k \Delta_{f}\left(m T_{s a m}+\tau_{s a m}-T_{G I 2}\right)}$,

and $\eta_{q M}$ 's, $q=8, \ldots, 11$, are independent, zero-mean, circularly-symmetric complex-Gaussian random variables with variance

$E\left\{\eta_{q M} \eta_{q M}^{*}\right\}=\mathrm{SNR}^{-1} \gamma^{-1}$

In (19), the signal-to-noise ratio (SNR) is computed by SNR = $P /\left(N_{0} W\right)$, and

$\gamma^{-1}=\sum_{m=1}^{M} g_{m} g_{m}^{*}$

For $\tau_{\text {sam }}>0$, the second subpreamble has a minor contribution 
to $\Xi_{10 M}$. We ignore this minor contribution in the derivation of (16) in order to keep the expression of $\Xi_{10 M}$ consistent to those of $\Xi_{8 M}$ and $\Xi_{9 M}$.

The probability of false alarm in a single comparison is defined as the probability that $\beta \mu_{9 M}>\left|\Xi_{9 M}\right|^{2}$.(or $\beta \mu_{10 M}>$ $\left.\left|\Xi_{10 M}\right|^{2}\right)$. The probability of miss is the probability that $\beta \mu_{11 M} \leq\left|\Xi_{11 M}\right|^{2}$. Conditioned on $\tau_{\text {sam }}$, the probability of false alarm in a single comparison, $P_{f a}(\cdot)$, and the probability of miss, $P_{m}(\cdot)$, are given by

$P_{f a}\left(\tau_{s a m}\right)=\operatorname{Pr}\left\{\beta\left|Y\left(\tau_{s a m}\right)+\eta_{f a 1}\right|^{2}>\left|Y\left(\tau_{s a m}\right)+\eta_{f a 2}\right|^{2}\right\}$

and

$P_{m}\left(\tau_{s a m}\right)=\operatorname{Pr}\left\{\beta\left|Y\left(\tau_{s a m}\right)+\eta_{m 1}\right|^{2}<\left|Z\left(\tau_{s a m}\right)+\eta_{m 2}\right|^{2}\right\}$,

respectively, where $\eta_{f a 1}, \eta_{f a 2}, \eta_{m 1}$ and $\eta_{m 2}$ are independent zero-mean complex-Gaussian random variables with variance $\mathrm{SNR}^{-1} \gamma^{-1}$. It is desirable to express $P_{f a}(\cdot)$ and $P_{m}(\cdot)$ as a function of $E_{d s} / N_{0}$ where $E_{d s}=\frac{1}{52} P T_{d s}$ is the data-symbol energy, $T_{d s}=3.2 \mu$ s being the duration of a data symbol excluding the cyclic prefix. Let

$\Omega=\gamma \cdot \mathrm{SNR}=\left(\frac{52 \gamma}{T_{d s} W}\right) \cdot \frac{E_{d s}}{N_{0}}$

Also let $2 \sigma_{\eta}^{2}=\Omega^{-1}$. Eqns. (21) and (22) can be expressed as

$P_{f a}\left(\tau_{s a m}\right)=\operatorname{Pr}\left\{\beta>\frac{\left|\frac{Y\left(\tau_{s a m}\right)}{\sigma_{\eta}}+\frac{\eta_{f a 2}}{\sigma_{\eta}}\right|^{2}}{\left|\frac{Y\left(\tau_{s a m}\right)}{\sigma_{\eta}}+\frac{\eta_{f a 1}}{\sigma_{\eta}}\right|^{2}}\right\}$

and

$P_{m}\left(\tau_{\text {sam }}\right)=\operatorname{Pr}\left\{\beta^{-1}>\frac{\left|\frac{Y\left(\tau_{s a m}\right)}{\sigma_{\eta}}+\frac{\eta_{m 1}}{\sigma_{\eta}}\right|^{2}}{\left|\frac{Z\left(\tau_{s a m}\right)}{\sigma_{\eta}}+\frac{\eta_{m 2}}{\sigma_{\eta}}\right|^{2}}\right\}$,

respectively. It is recognized that

$$
\begin{aligned}
& \left|\frac{Y\left(\tau_{\text {sam }}\right)}{\sigma_{\eta}}+\frac{\eta_{f a 2}}{\sigma_{\eta}}\right|^{2} /\left|\frac{Y\left(\tau_{s a m}\right)}{\sigma_{\eta}}+\frac{\eta_{f a 1}}{\sigma_{\eta}}\right|^{2} \\
& \sim F_{2,2}^{\prime \prime}\left(2 \Omega\left|Y\left(\tau_{\text {sam }}\right)\right|^{2}, 2 \Omega\left|Y\left(\tau_{s a m}\right)\right|^{2}\right)
\end{aligned}
$$

and

$$
\begin{aligned}
& \left|\frac{Y\left(\tau_{\text {sam }}\right)}{\sigma_{\eta}}+\frac{\eta_{m 1}}{\sigma_{\eta}}\right|^{2} /\left|\frac{Z\left(\tau_{\text {sam }}\right)}{\sigma_{\eta}}+\frac{\eta_{m 2}}{\sigma_{\eta}}\right|^{2} \\
& \sim F_{2,2}^{\prime \prime}\left(2 \Omega\left|Y\left(\tau_{s a m}\right)\right|^{2}, 2 \Omega\left|Z\left(\tau_{\text {sam }}\right)\right|^{2}\right)
\end{aligned}
$$

where $\sim$ denotes "follows the distribution of" and $F_{2,2}^{\prime \prime}\left(\lambda_{1}, \lambda_{2}\right)$ is the doubly noncentral $F$-distribution with 2,2 degrees of freedom and noncentrality parameters $\lambda_{1}, \lambda_{2}$ [9]. It is known that [10]

$$
\begin{array}{r}
\operatorname{Pr}\left\{f>F_{2,2}^{\prime \prime}\left(\lambda_{1}, \lambda_{2}\right)\right\}=Q\left(\sqrt{\frac{f \lambda_{2}}{1+f}}, \sqrt{\frac{\lambda_{1}}{1+f}}\right) \\
-\frac{1}{1+f} e^{-\frac{f \lambda_{2}+\lambda_{1}}{2(1+f)}} I_{0}\left(\frac{\sqrt{f \lambda_{1} \lambda_{2}}}{1+f}\right)
\end{array}
$$

where $Q(\cdot$,$) is Marcum's Q$ function and $I_{0}(\cdot)$ is the modified Bessel function of the first kind and order zero. Applying the relationships (26) and (27) to (24) and (25), respectively, and making use of the result (28), we obtain closed-form expressions of $P_{f a}\left(\tau_{s a m}\right)$ and $P_{m}\left(\tau_{s a m}\right)$. The probability of synchronization failure, $P_{f a i l}$, is hence given by

$P_{\text {fail }}=\frac{1}{T_{\text {sam }}} \int_{-T_{\text {sam }} / 2}^{T_{\text {sam }} / 2} P_{\text {fail }}\left(\tau_{\text {sam }}\right) d \tau_{\text {sam }}$

where

$P_{f a i l}\left(\tau_{s a m}\right)=1-\left[1-P_{f a}\left(\tau_{s a m}\right)\right]^{2}+\left[1-P_{f a}\left(\tau_{s a m}\right)\right]^{2} P_{m}\left(\tau_{s a m}\right)$

Numerical computation of the integration in (29) can be readily accomplished by Simpson's rule.

\section{APPENDIX III. SIMULATION CONDITIONS}

To evaluate the goodness of a set of correlator coefficients $g_{m}, m=1,2, \cdots, M$, one needs to simulate $P_{\text {fail }}$ for a multipath Rician fading channel. We adopt the conditions similar to those used in [5] to perform simulation.

Since the signal is bandlimited, it is known that the channel can be modeled by a tapped delay-line model [11, pp. 795]. The channel impulse response, $h(t)$, is given by

$h(t)=A e^{j \phi} \delta(t)+\sum_{\ell=0}^{L-1} \alpha_{\ell} e^{j \theta_{\ell}} \delta\left(t-\ell T_{B}\right)$

where (a) $A$ and $\phi$ are the deterministic gain and random phase, respectively, of the direct path, (b) $\alpha_{\ell}$ and $\theta_{\ell}$ are the gain and phase of the $\ell$ th faded path, modeled by, respectively, a Rayleigh-distributed random variable with a 
variance $\overline{\alpha_{\ell}^{2}}$ and a uniform random variable over $[0,2 \pi$ ), (c) $L$ is the number of resolvable paths or the number of taps in the channel model, and (d) $T_{B}$ is the inverse of the signal bandwidth. We use $T_{B}=50 \mathrm{~ns}$ since the signal occupies approximately $20 \mathrm{MHz}[1]$. Note that $\alpha_{\ell}$ and $\theta_{\ell}, \quad \ell=$ $0,1, \cdots, L-1$, are statistically independent. The Rician factor, $K$, is given by $K=A^{2} / \sum_{\ell=0}^{L-1} \overline{\alpha_{\ell}^{2}}$. The channel dispersion is modeled by an exponential function, which is appropriate for modeling indoor radio channels in wireless LANs [12]. It is assumed that the channel has a unity gain so that $A^{2}+$ $\sum_{\ell=0}^{L-1} \overline{\alpha_{\ell}^{2}}=1$. We calculate $A$ and $\overline{\alpha_{\ell}^{2}}$ by

$A=\sqrt{\frac{K}{K+1}}$

and

$\overline{\alpha_{\ell}^{2}}=\frac{1}{K+1} \int_{\ell T_{B}}^{(\ell+1) T_{B}} \tau_{r m s}^{-1} e^{-\tau / \tau_{r m s}} d \tau$

where $\tau_{r m s}$ is the RMS delay spread of the Rayleigh-faded components.

In the simulation, we considered that $\tau_{r m s}=50 \mathrm{~ns}$ and $K \in$ $\{-\infty \mathrm{dB}, 6 \mathrm{~dB}\}$. We set $L=10$ for maintaining adequate accuracy in the generation of the exponential channel dispersion without incurring excessive simulation time. In each simulation run, a realization of $h(t)$ [given by (31)] was substituted into (8) to yield $r(t)$. The sequence of received signal samples, $\left\{r_{n}\right\}$ was then obtained from $r(t)$ by (10) with a randomly generated $\tau_{\text {sam }}$. Afterwards, the sequence of correlation outputs $\left\{\Xi_{n}\right\}$ was computed. The synchronization procedure was then carried out. The declared synchronization point was compared with the time instant of the end of the first subpreamble. If the declared synchronization point was different from the actual end of the first subpreamble by more than a given tolerance, a synchronization failure was counted. Since OFDM is rather robust to small time offsets as a result of the inclusion of cyclic prefix (800ns) in the data format [13], in the simulation a tolerance of $\pm 300 \mathrm{~ns}$ was given. The value of $P_{\text {fail }}$ was computed as the ratio of the number of synchronization failures to the total number of simulation runs.

\section{REFERENCES}

[1] IEEE Computer Society, IEEE Std 802.11a-1999, 30 Dec. 1999.

[2] T. Saito, I. Tomoda, Y. Takabatake, K. Teramoto and K. Fujimoto, "Wireless gateway for wireless home AV network and its implementation," IEEE Trans. Consumer Electronics, vol. 47, pp. 496501, Aug. 2001.

[3] A. Doufexi, D. Redmill, D. Bull and A. Nix, "MPEG-2 video transmission using the HIPERLAN/2 WLAN standard," IEEE Trans. Consumer Electronics, vol. 47, pp. 354-363, Aug. 2001.

[4] P. H. Moose, "A technique for orthogonal frequency division multiplexing frequency offset correction," IEEE Trans. Commun., vol. 42, pp. 2908-2914, Oct. 1994.
[5] K. W. Yip, T. S. Ng and Y. C. Wu, "Impacts of multipath fading on the timing synchronization of IEEE 802.11a wireless LANs," Proc. IEEE International Conference on Communications (ICC 2002), pp. 517521, Apr. 28 - May 2, 2002.

[6] R. J. C. Bultitude, "Measurement, characterization and modeling of indoor $800 / 900 \mathrm{MHz}$ radio channels for digital communications," IEEE Commun. Mag., vol. 25, no. 6, pp. 5-12, Jun. 1987.

[7] T. S. Rappaport and C. D. McGillem, "UHF fading in factories," IEEE J. Select. Areas Commun., vol. 7, pp. 40-48, Jan. 1989

[8] S.-C. Kim, H. L. Bertoni and M. Stern, "Pulse propagation characteristics at $2.4 \mathrm{GHz}$ inside buildings," IEEE Trans. Veh. Technol., vol. 45, pp. 579-592, Aug. 1996.

[9] N. L. Johnson, S. Kotz and N. Balakrishnan, Continuous Univariate Distributions, vol. 2, 2nd ed., ch. 30, New York: Wiley, 1995.

[10] R. Price, "Some non-central $F$-distributions expressed in closed form," Biometrika, vol. 51, pts. 1,2, pp. 107-122, Jun. 1964.

[11] J. G. Proakis, Digital Communications, 3rd ed., New York: McGrawHill, 1995.

[12] J. Hansen and $M$. Nold, "Analytic calculation of the power delay profile for single room wireless LAN environments," Proc. IEEE GLOBECOM 2000, pp. 98-102, Nov. 27 - Dec. 1, 2000.

[13] R. van Nee and R. Prasad, OFDM for Wireless Multimedia Communications, ch. 4.4, Boston: Artech-House, 2000.

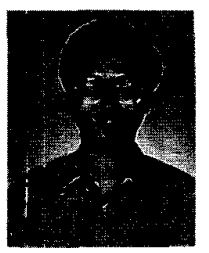

Kun-Wah Yip (M'96) received the B.Eng. (Hons) and Ph.D. degrees in electrical engineering from The University of Bradford, UK, in 1991 and The University of Hong Kong in 1995, respectively.

From 1995 to 1998 , he was a research associate and then a postdoctoral fellow at The University of Hong Kong. Currently, he is a research assistant professor at the same university. His research interest is on WLANs, WPANs, communication circuits, spreadspectrum techniques, OFDM, and efficient simulation techniques for communication systems.

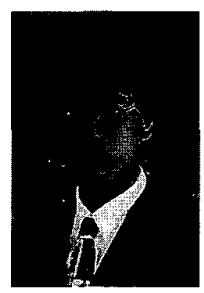

Yik-Chung $\mathrm{Wu}$ (M'02) received the B.Eng.(Hons) and M.Phil. degrees in Electronic Engineering from The University of Hong Kong in 1998 and 2001, respectively. After graduation, he was a research assistant at the same university. Currently, he is pursuing his Ph.D. degree at the Texas A\&M University, TX. His research interests are in digital signal processing with applications to communication systems and software radio, and in space-time processing.

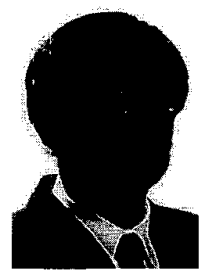

Tung-Sang Ng (S'74-M'78-SM'90-F'03) received the B.Sc.(Eng.) degree from the University of Hong Kong in 1972, and the M.Eng.Sc. and Ph.D. degrees from the University of Newcastle, Australia, in 1974 and 1977, respectively, all in electrical engineering.

$\mathrm{He}$ worked for BHP Steel International and The University of Wollongong, Australia, after graduation for 14 years and returned to Hong Kong in 1991, taking up the position of Professor and Chair of Electronic Engineering. $\mathrm{He}$ is Head of Department of Electrical and Electronic Engineering since 2000. His current research interests include wireless communication systems, spread spectrum techniques, CDMA and digital signal processing. He was the General Chair of ISCAS'97 and the VP-Region 10 of IEEE CAS Society $(1999$ \& 2000). He was an Executive Committee Member and a Board Member of the IEE Informatics Divisional Board (19992001) and was an ordinary member of IEE Council (1999-2001). He hás published over 170 International journal and conference papers. 